\title{
Author Correction: Long-range chiral exchange interaction in synthetic antiferromagnets
}

Dong-Soo Han, Kyujoon Lee, Jan-Philipp Hanke iD, Yuriy Mokrousov D, Kyoung-Whan Kim (D), Woosuk Yoo,

Youri L. W. van Hees, Tae-Wan Kim, Reinoud Lavrijsen, Chun-Yeol You D, Henk J. M. Swagten, Myung-Hwa Jung (D) and Mathias Kläui(iD

Correction to: Nature Materials https://doi.org/10.1038/s41563-019-0370-z, published online 3 June 2019.

In the version of this Article originally published, the sentence 'D.-S.H. wrote the paper with K.L., J.H. and M.K.' in the author contributions was incorrect; it should have read 'D.-S.H. wrote the paper with K.L., J.H., M.-H.J. and M.K.' This has been corrected in the online versions of the Article.

Published online: 25 June 2019

https://doi.org/10.1038/s41563-019-0442-0 